QUARTERLY OF APPLIED MATHEMATICS

VOLUME LXX, NUMBER 1

MARCH 2012, PAGES 1-24

S 0033-569X(2011)01240-2

Article electronically published on September 15, 2011

\title{
MATHEMATICAL MODELLING OF AVASCULAR ELLIPSOIDAL TUMOUR GROWTH
}

\author{
BY \\ G. DASSIOS (Department of Chemical Engineering, University of Patras, GR 265 04, Patras, Greece \\ and ICE-HT/FORTH, Greece), \\ F. KARIOTOU (Department of Chemical Engineering, University of Patras, GR 265 04, Patras, \\ Greece), \\ M. N. TSAMPAS (Department of Chemical Engineering, University of Patras, GR 265 04, Patras, \\ Greece), \\ AND \\ B. D. SLEEMAN (School of Mathematics, University of Leeds, Leeds, LS2 9JT, United Kingdom)
}

\begin{abstract}
Breast cancer is the most frequently diagnosed cancer in women. From mammography, Magnetic Resonance Imaging (MRI), and ultrasonography, it is well documented that breast tumours are often ellipsoidal in shape. The World Health Organisation (WHO) has established a criteria based on tumour volume change for classifying response to therapy. Typically the volume of the tumour is measured on the hypothesis that growth is ellipsoidal. This is the Calliper method, and it is widely used throughout the world. This paper initiates an analytical study of ellipsoidal tumour growth based on the pioneering mathematical model of Greenspan. Comparisons are made with the more commonly studied spherical mathematical models.
\end{abstract}

1. Tumour biology. Cell proliferation is normally a highly regulated process, such that only the required numbers of cells populate a given tissue. If control of proliferation is altered or lost, cells may continue to divide leading to an abnormal mass of tissue - a tumour. The most common cause of tumours is genetic mutation resulting in uncontrolled cell division. Although a single mutation can account for this loss of control, it is far more common for a series of mutations in a number of genes to accumulate, eventually resulting in loss of proliferative control. This increased cell mass can be due to increased cell division or a decrease in programmed cell death, which normally occurs as part of limiting cell numbers, or a combination of both.

Two classes of genes that are commonly mutated in tumours are oncogenes and tumour suppressors. Oncogenes are mutated forms of proto-oncogenes, which normally encode

Received March 18, 2010.

2010 Mathematics Subject Classification. Primary 92C05, 92C50.

(C)2011 Brown University 
proteins involved in growth promoting signal transduction and mitogenesis. Oncogenes are more active, hence increasing the rate of proliferation. Tumour suppressor genes, as their name suggests, are normally involved in slowing cell growth and division; mutations release this control, again increasing proliferation. Mutations in either class of gene, or in both, result in reduced control of cell growth and division, giving these cells a growth advantage over neighbouring healthy cells and leading to the development of a tumour.

Tumours can be benign or malignant, dependent on their aggressiveness. A benign tumour stays as a noninvasive cluster, without spreading into its surroundings. The margin of the tumour is very distinct and the whole tumour can usually be removed by surgery. By aquiring more mutations, a benign tumour can become malignant. Malignant tumours grow aggressively and invade into surrounding tissue. Tumour cells that break away from the parent tumour, and move via lymphatic or blood vessels to a distant site to form secondary tumours or metastases, are characteristic of a malignant tumour.

Most tumours start as a small mass of rapidly proliferating cells, where nutrients and oxygen are acquired by passive diffusion from the surrounding tissues, the size of the tumour is limited to about $2 \mathrm{~mm}$ in diameter and tumours can stay in this diffusion limited state, where cell proliferation is balanced by cell death, for months or even years. It was over thirty years ago that Judah Folkman [10] first proposed that in order to develop beyond this dormant state, the tumour must induce the growth of new blood vessels in order to supply the increasing metabolic demands. At that time, the mechanism of new vessel growth, called angiogenesis, was not known, but Folkman suggested that the switch in a tumour towards a pro-angiogenic state is a specific stage in tumour development. Once the tumour becomes vascularised, diffusion no longer limits size, and the tumour can grow and develop.

It is now known that control of angiogenesis is orchestrated by a large number of pro-angiogenic and anti-angiogenic factors, and it is the shift in balance from anti- to pro-angiogenic that elicits the so-called angiogenic switch and induces growth of blood vessels towards the tumour. Many factors contribute to the switch towards angiogenesis, one of which is oxygen deficiency within the tumour. Tissues deprived of oxygen become hypoxic, and express a range of factors to help them survive, some of which are proangiogenic factors, driving the growth of new blood vessels towards that tissue.

This whole orchestration of complex events leads to a micro-vascular structure that eventually reaches and penetrates the tumor, vastly improving its blood supply and allowing for rapid and unconstrained growth. For an up-to-date account of the biochemistry of tumour angiogenesis, we refer to Plank and Sleeman [14] as well as the references [3], [13] and [16.

In this paper we consider the growth of avascular tumours, the first step being in understanding the growth of complex processes involved in angiogenesis and vascular structures. There exist in the literature several mathematical models of avascular tumour growth. These include (i) models that describe continuum cell populations and their growth by considering the interactions between cell density and the chemical species that provide nutrients as well as inhibitors, (ii) models that describe mechanical interactions between tumour cells and their surroundings and (iii) individual cell based models that allows one to track cells in both space and time. From in vitro experiments and 
some observed in vivo studies, it is known that avascular tumours may grow as symmetric spheroids wherein growth is essentially radial. In this situation many of the above mathematical models admit to analytical treatment and enable one to determine cell movement, track the spheroidal boundary and to assess the roles of growth inhibitors and growth promoters. The stability of tumour growth can also be analysed; see [12] for a review and cited references.

A solid mass growing in healthy tissue produces stress. Models [11] have been developed to study this form of mechanical stress in which the tumour deforms the surrounding tissue due to the stress it imposes on the environment, and the environment in turn alters tumour growth dynamics. In these models, tumour growth inhibition depends on the stiffness of the surrounding environment. In an in vivo setting this corresponds to the stiffness of the extracellular matrix environment. In an in vitro setting, this corresponds to the stiffness of the agarose gel. The effects of physical confinement on tumour growth have been studied experimentally. In [8], human colon adenocarcinoma cells were grown in cylindrical glass tubes with a radius that was much smaller than the length of the tube. It was found that cell aggregates in $0.7 \%$ gel placed in a capillary tube grew to take on an ellipsoidal shape driven by the geometry of the capillary tube. The same cells grown outside a capillary tube developed into a spherical shape. This experiment highlights that geometric confinement alters the shape and growth dynamics of a developing tumour. Mathematical models which treat avascular tumours as visco-elastic materials and discuss the effects of mechanical stress are considered in [4, 15].

Breast cancer is the most frequently diagnosed cancer in women. From mammography, magnetic resonance imaging (MRI) and ultrasonography it is well documented that breast tumours are often ellipsoidal in shape. Indeed the World Health Organisation (WHO) established in 1979 criteria based on tumour volume change for classifying response to therapy as progressive disease, partial recovery or no change. Typically the volume of the tumour is measured on the hypothesis that growth is ellipsoidal. This is the socalled calliper method and is widely used throughout the world in assessing and grading gliomas. See [2, 5, 17.

In this paper we initiate an analytical study of ellipsoidal tumour growth based on the pioneering mathematical model of avascular tumour growth due to Greenspan [6, 7, 15].

In section 2 we formulate the mathematical model in terms of ellipsoidal geometry and explicitly solve for the pressure field and nutrient concentration within a growing avascular ellipsoidal tumour made up of a live cell layer, a quiescent layer and a necrotic core. Because the analysis depends extensively on the use of Lamé functions, their relevant properties together with a description of ellipsoidal coordinates are outlined in Appendix A. Section 3 contains a resumé of the well-known spherical tumour problem and emphasises the modelling differences with the Greenspan model. In section 4 we carry out a number of numerical simulations. The paper concludes in section 5 with a discussion of the results.

2. The mathematical model. The tumour is assumed to have a three-layer structure consisting of a thin outer layer of live proliferating cells that envelops an inner layer of quiescent live but not proliferating cells which in turn envelops a large necrotic core of 
dead cells and debris. Cells proliferate as long as the available concentration of nutrient supply, denoted by $\sigma(\mathbf{r}, t)$, remains above a critical level $\sigma_{1}$. A cell dies due to apoptosis or otherwise when $\sigma$ falls below a critical level $\sigma_{2}$. In the quiescent region, nutrient supply varies over the interval $\sigma_{2} \leq \sigma \leq \sigma_{1}$. The characteristic thickness $s$ of the layer of live proliferating cells depends on $\sigma_{1}$ and the value of $\sigma$ at the outer surface of the tumour.

It is assumed that the tumour boundaries evolve as members of the confocal ellipsoidal family with foci $\left( \pm h_{2}, 0,0\right),\left( \pm h_{3}, 0,0\right),\left(0, \pm h_{1}, 0\right)$ in accordance with the ellipsoidal coordinate system defined by

$$
\begin{aligned}
x_{1} & =\frac{\rho \mu \nu}{h_{2} h_{3}}, \\
x_{2} & =\frac{\sqrt{\rho^{2}-h_{3}^{2}} \sqrt{\mu^{2}-h_{3}^{2}} \sqrt{h_{3}^{2}-\nu^{2}}}{h_{1} h_{3}}, \\
x_{3} & =\frac{\sqrt{\rho^{2}-h_{2}^{2}} \sqrt{h_{2}^{2}-\mu^{2}} \sqrt{h_{2}^{2}-\nu^{2}}}{h_{1} h_{2}},
\end{aligned}
$$

where $(\rho, \mu, \nu)$ and $\left(x_{1}, x_{2}, x_{3}\right)$ are the ellipsoidal and Cartesian coordinates, respectively. The basic ellipsoid is given, in Cartesian coordinates, by

$$
\frac{x_{1}^{2}}{\alpha_{1}^{2}}+\frac{x_{2}^{2}}{\alpha_{2}^{2}}+\frac{x_{3}^{2}}{\alpha_{3}^{2}}=1, \quad 0<\alpha_{3}<\alpha_{2}<\alpha_{1}<+\infty,
$$

where the semi-focal distances are given by

$$
h_{1}^{2}=\alpha_{2}^{2}-\alpha_{3}^{2}, h_{2}^{2}=\alpha_{1}^{2}-\alpha_{3}^{2}, h_{3}^{2}=\alpha_{1}^{2}-\alpha_{2}^{2} .
$$

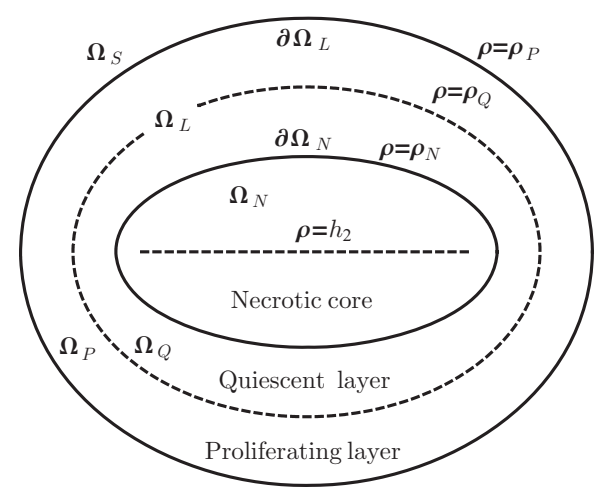

FIG. 1. Ellipsoidal tumour with the 3-layer structure of necrotic core, quiescent layer, and proliferating layer.

Let $\Omega_{N}$ denote the ellipsoid occupied by the tumour's necrotic core. Similarly, let $\Omega_{Q}, \Omega_{P}$ denote the ellipsoidal shells occupied by the quiescent and proliferating cells, respectively. The exterior of the ellipsoidal tumour is denoted by $\Omega_{S}$. The boundaries of these regions, denoted by $\partial \Omega_{N}, \partial \Omega_{Q}, \partial \Omega_{P}$, correspond to the coordinate values $\rho=\rho_{N}, \rho=\rho_{Q}, \rho=\rho_{P}$, respectively. Next, let $P(\mathbf{r})$ denote the pressure field and $\sigma(\mathbf{r})$ 
the nutrient concentration at the point $\mathbf{r}=(\rho, \mu, \nu)$. Finally, the threshold values $\sigma_{1}$ and $\sigma_{2}$ of $\sigma(\mathbf{r})$ specify the above ellipsoidal regions as

$$
\begin{aligned}
& \Omega_{N}=\left\{(\rho, \mu, \nu): h_{2} \leq \rho<\rho_{N}, \sigma(\mathbf{r})<\sigma_{2}\right\}, \\
& \Omega_{Q}=\left\{(\rho, \mu, \nu): \rho_{N}<\rho<\rho_{Q}, \sigma_{2}<\sigma(\mathbf{r})<\sigma_{1}\right\}, \\
& \Omega_{P}=\left\{(\rho, \mu, \nu): \rho_{Q}<\rho<\rho_{P}, \sigma(\mathbf{r})>\sigma_{1}\right\}, \\
& \Omega_{S}=\left\{(\rho, \mu, \nu): \rho>\rho_{P}, \sigma_{1}<\sigma(\mathbf{r})<\sigma_{\infty}\right\},
\end{aligned}
$$

where $\sigma_{\infty}$ is the ambient nutrient concentration outside the tumour.

Regarding the tumour as an incompressible fluid, local changes in the cell population due to proliferation and apoptsis will induce motion of the neighboring cells. Denote by $S=S(\sigma)$ the cell loss rate at a point inside the tumour and denote the cell velocity by $\mathbf{q}(\mathbf{r}, t)$, where $\mathbf{r}$ is the position vector in ellipsoidal coordinates.

By applying the law of conservation of mass one arrives at the following equation for q:

$$
\nabla \cdot \mathbf{q}=-S(\sigma)
$$

The next step is to introduce Darcy's law which relates internal pressure $p$ to the velocity $\mathbf{q}(\mathbf{r}, t)$ by

$$
\mathbf{q}(\mathbf{r}, t)=-\nabla p
$$

By eliminating $\mathbf{q}$ between these equations we get

$$
\triangle p=S(\sigma) .
$$

Reaction-diffusion kinetics are used to model the evolution of the nutrient $\sigma$. It is known that a typical chemical diffusion time scale is much shorter than a typical tumour cell doubling time so that, as the tumour grows, $\sigma$ rapidly diffuses throughout the new volume. As a consquence it can be assumed that $\sigma$ is in a diffusive steady state. In other words, $\sigma$ is governed by

$$
\triangle \sigma=0 .
$$

The cell loss rate $S$ is modelled in the following way. Cell loss due to apoptosis is restricted to the proliferating and quiescent region and is assumed to occur at the constant rate $S_{1}$. Cell loss due to necrosis is assumed to occur at the constant rate $S_{2}$. In terms of the Heaviside step function $H$ we can write $S$ in the concise form

$$
S(\sigma)=S_{1} H\left(|\mathbf{r}|-\left|\mathbf{r}_{N}\right|\right)+S_{2} H\left(\left|\mathbf{r}_{N}\right|-|\mathbf{r}|\right),
$$

where $\mathbf{r}_{N}$ denotes a point on the surface of the necrotic region.

In accordance with Greenspan [6, 7] we have the following relations holding on the surface of the tumour:

$$
\begin{aligned}
\mathbf{q} \cdot \hat{\mathbf{n}} & =-\hat{\mathbf{n}} \cdot \nabla p+\frac{\beta}{d_{t}} s, \\
\mathbf{q} \times \hat{\mathbf{n}} & =-\hat{\mathbf{n}} \times \nabla p
\end{aligned}
$$

together with the nutrient boundary conditions

$$
\hat{\mathbf{n}} \cdot \nabla \sigma=\frac{\gamma}{k} s
$$


In equations (12) and (13), the velocity $\mathbf{q}$ is given by

$$
\mathbf{q}(\mathbf{r}, t)=h_{\rho} \frac{d \rho}{d t} \hat{\boldsymbol{\rho}}+h_{\mu} \frac{d \mu}{d t} \hat{\boldsymbol{\mu}}+h_{\nu} \frac{d \nu}{d t} \hat{\boldsymbol{\nu}}
$$

on $\rho=\rho_{p}$ and the metric coefficients $h_{i}, i=\rho, \mu, \nu$ are defined in Appendix A.

In addition $\sigma$ is assumed continuous together with its normal derivative across the interior surfaces of the tumour. We also assume that the surrounding medium is large in comparison with the tumour size and that there is a constant supply of nutrient, i.e.,

$$
\sigma \rightarrow \sigma_{\infty}, \text { as }|\mathbf{r}| \rightarrow \infty
$$

In equations (12) and (14), $\beta$ is the rate of mass/volume production, $d_{t}$ the mass density of the tumour colony, $\gamma$ is the rate of mass/volume consumption, $k$ the diffusion constant and $s$ is the local thickness of the live outer layer of proliferating cells.

On the outer boundary $\partial \Omega_{P}$ of the tumour we assume that the pressure $p$ satisfies the Young-Laplace relation [1]

$$
p_{\text {in }}-p_{\text {out }}=a \kappa .
$$

This relation states that the pressure difference across $\partial \Omega_{P}$ is proportional to the mean curvature $\kappa$, which is the energy needed to maintain the intercellular bonds which exist on $\partial \Omega_{P}$. On the boundary $\partial \Omega_{N}$ separating the necrotic and live layers we assume continuity of the nutrient together with continuity of the pressure and its normal derivative.

2.1. The nutrient concentration problem. Let $\sigma_{N}(\mathbf{r}), \sigma_{L}(\mathbf{r})$ and $\sigma_{S}(\mathbf{r})$ denote the nutrient concentrations at the point $\mathbf{r}$ of $\Omega_{N}, \Omega_{L}=\Omega_{Q} \cup \Omega_{P}$ and $\Omega_{S}$ respectively. Then from (10) we can write

$$
\Delta \sigma_{i}(\mathbf{r})=0, \mathbf{r} \in \Omega_{i}, i=N, L, S .
$$

On the boundary $\partial \Omega_{N}$ we have

$$
\sigma_{N}\left(\mathbf{r}_{N}\right)=\sigma_{L}\left(\mathbf{r}_{N}\right)
$$

while on the exterior boundary $\partial \Omega_{L}$ we have

$$
\sigma_{L}\left(\mathbf{r}_{P}\right)=\sigma_{S}\left(\mathbf{r}_{P}\right),
$$

together with

$$
\hat{\boldsymbol{\rho}} \cdot \nabla \sigma_{L}\left(\mathbf{r}_{P}\right)=\hat{\boldsymbol{\rho}} \cdot \nabla \sigma_{S}\left(\mathbf{r}_{P}\right),
$$

where $\hat{\boldsymbol{\rho}}$ is the unit outward normal at the point $\mathbf{r}_{P}=\left(\rho_{P}, \mu, \nu\right)$ of the ellipsoidal outer boundary. Note also that from (14) we have

$$
\hat{\boldsymbol{\rho}} \cdot \nabla \sigma_{L}\left(\mathbf{r}_{P}\right)=\frac{\gamma}{k} s\left(\mathbf{r}_{P}\right) .
$$

The local thickness $s\left(\mathbf{r}_{\mathbf{P}}\right)$ of the tumour's outer live cell layer, considered small compared to $\Omega_{N}$, is given by the expression

$$
s\left(\mathbf{r}_{P}\right)=h_{\rho}\left(\mathbf{r}_{P}\right)\left(\rho_{P}-\rho_{Q}\right) .
$$

We now assume that the nutrient concentration $\sigma$ is expressible in terms of ellipsoidal harmonics as

$$
\sigma_{S}(\mathbf{r})=\sigma_{L}(\mathbf{r})=\sum_{n=0}^{\infty} \sum_{m=1}^{2 n+1}\left[a_{n}^{m}+b_{n}^{m}(2 n+1) I_{n}^{m}(\rho)\right] E_{n}^{m}(\rho) E_{n}^{m}(\mu) E_{n}^{m}(\nu),
$$


for $\rho>\rho_{N}$ and

$$
\sigma_{N}(\mathbf{r})=\sum_{n=0}^{\infty} \sum_{m=1}^{2 n+1} c_{n}^{m} E_{n}^{m}(\rho) E_{n}^{m}(\mu) E_{n}^{m}(\nu),
$$

for $h_{2} \leq \rho<\rho_{N}$. Here $E_{n}^{m}(x)$ and $I_{n}^{m}(x)$ are the Lamé functions and the elliptic integral of degree $n$ and order $m$ and are connected to each other by

$$
I_{n}^{m}(\rho) \equiv \int_{\rho}^{\infty} \frac{d t}{\left[E_{n}^{m}(t)\right]^{2} \sqrt{\left(t^{2}-h_{2}^{2}\right)\left(t^{2}-h_{3}^{2}\right)}} .
$$

In the subsequent analysis we shall explicitly use only the following Lamé polynomials:

$$
\begin{aligned}
& E_{0}^{1}(x)=1, \\
& E_{2}^{1}(x)=x^{2}+\Lambda-\alpha_{1}^{2}, \\
& E_{2}^{2}(x)=x^{2}+\Lambda^{\prime}-\alpha_{1}^{2},
\end{aligned}
$$

where $\Lambda, \Lambda^{\prime}$ are the roots of the quadratic equation

$$
\sum_{i=1}^{3} \frac{1}{\Lambda-\alpha_{i}^{2}}=0
$$

From the definition of $h_{\rho}$ it can be shown that

$$
\begin{aligned}
{\left[h_{\rho}\left(\rho_{p}\right)\right]^{2}=} & \frac{1}{\left(\rho_{p}^{2}-h_{3}^{2}\right)\left(\rho_{p}^{2}-h_{2}^{2}\right)} \\
& \times\left[E_{2}^{1}\left(\rho_{p}\right) E_{2}^{2}\left(\rho_{p}\right) S_{0}^{1}(\mu, \nu)-\frac{E_{2}^{2}\left(\rho_{p}\right)}{\Lambda-\Lambda^{\prime}} S_{2}^{1}(\mu, \nu)+\frac{E_{2}^{1}\left(\rho_{p}\right)}{\Lambda-\Lambda^{\prime}} S_{2}^{2}(\mu, \nu)\right],
\end{aligned}
$$

where

$$
\begin{aligned}
& S_{0}^{1}(\mu, \nu)=1, \\
& S_{2}^{1}(\mu, \nu)=\left(\mu^{2}+\Lambda-a_{1}^{2}\right)\left(\nu^{2}+\Lambda-a_{1}^{2}\right), \\
& S_{2}^{2}(\mu, \nu)=\left(\mu^{2}+\Lambda^{\prime}-a_{1}^{2}\right)\left(\nu^{2}+\Lambda^{\prime}-a_{1}^{2}\right) .
\end{aligned}
$$

With these observations and using the boundary conditions together with the orthogonality of the Lamé polynomials we obtain

$$
\begin{array}{rc}
\sigma_{S}(\mathbf{r})= & \sigma_{L}(\mathbf{r})=\left[\sigma_{\infty}-\left(\sigma_{\infty}-\sigma_{2}\right) \frac{I_{0}^{1}(\rho)}{I_{0}^{1}\left(\rho_{N}\right)}\right] \\
- & -\left[\frac{\left(\sigma_{\infty}-\sigma_{2}\right)}{I_{0}^{1}\left(\rho_{N}\right)} \frac{I_{2}^{1}(\rho) E_{2}^{1}(\rho)}{(3 / 2 \pi) I_{2}^{1}\left(\rho_{P}\right) E_{2}^{1}\left(\rho_{P}\right) V\left(\rho_{P}\right)-1}\right] \frac{S_{2}^{1}(\mu, \nu)}{\Lambda-\Lambda^{\prime}} \\
+ & \left.+\frac{\left(\sigma_{\infty}-\sigma_{2}\right)}{I_{0}^{1}\left(\rho_{N}\right)} \frac{I_{2}^{2}(\rho) E_{2}^{2}(\rho)}{(3 / 2 \pi) I_{2}^{2}\left(\rho_{P}\right) E_{2}^{2}\left(\rho_{P}\right) V\left(\rho_{P}\right)-1}\right] \frac{S_{2}^{2}(\mu, \nu)}{\Lambda-\Lambda^{\prime}}
\end{array}
$$

and

$$
\begin{array}{rc}
\sigma_{N}(\mathbf{r})= & \sigma_{2} \\
& -\left[\frac{\left(\sigma_{\infty}-\sigma_{2}\right)}{I_{0}^{1}\left(\rho_{N}\right)} \frac{I_{2}^{1}\left(\rho_{N}\right) E_{2}^{1}(\rho)}{(3 / 2 \pi) I_{2}^{1}\left(\rho_{P}\right) E_{2}^{1}\left(\rho_{P}\right) V\left(\rho_{P}\right)-1}\right] \frac{S_{2}^{1}(\mu, \nu)}{\Lambda-\Lambda^{\prime}} \\
& +\left[\frac{\left(\sigma_{\infty}-\sigma_{2}\right)}{I_{0}^{1}\left(\rho_{N}\right)} \frac{I_{2}^{2}\left(\rho_{N}\right) E_{2}^{2}(\rho)}{(3 / 2 \pi) I_{2}^{2}\left(\rho_{P}\right) E_{2}^{2}\left(\rho_{P}\right) V\left(\rho_{P}\right)-1}\right] \frac{S_{2}^{2}(\mu, \nu)}{\Lambda-\Lambda^{\prime}}
\end{array}
$$

where

$$
\sigma_{2}=\sigma_{\infty}-\frac{\gamma}{\kappa} \frac{\left(\rho_{P}-\rho_{Q}\right)\left(\rho_{P}^{2}+\Lambda-\alpha_{1}^{2}\right)\left(\rho_{P}^{2}+\Lambda^{\prime}-\alpha_{1}^{2}\right) I_{0}^{\prime}\left(\rho_{N}\right)}{\sqrt{\left(\rho_{P}^{2}-h_{3}^{2}\right)} \sqrt{\left(\rho_{P}^{2}-h_{2}^{2}\right)}}
$$


In (28) and (29) the volume $V\left(\rho_{P}\right)$ of the ellipsoid $\rho=\rho_{P}$ is given by

$$
V(\rho)=\frac{4 \pi}{3} \rho \sqrt{\left(\rho^{2}-h_{3}^{2}\right)\left(\rho^{2}-h_{2}^{2}\right)} .
$$

In terms of the outer boundary $\rho_{P}$ we can use

$$
\frac{\partial \sigma_{L}\left(\mathbf{r}_{P}\right)}{\partial \rho}=\frac{\gamma}{k} h_{\rho}\left(\rho_{P}\right) s\left(\rho_{P}\right)
$$

to show that

$$
\left(\rho_{P}-\rho_{Q}\right) I_{0}^{1}\left(\rho_{N}\right)=\frac{k\left(\sigma_{\infty}-\sigma_{2}\right)}{\gamma} \frac{\sqrt{\left(\rho_{P}^{2}-h_{3}^{2}\right)\left(\rho_{P}^{2}-h_{2}^{2}\right)}}{\left(\rho_{P}^{2}+\Lambda-\alpha_{1}^{2}\right)\left(\rho_{P}^{2}+\Lambda^{\prime}-\alpha_{1}^{2}\right)} .
$$

If we set

$$
\sigma_{1}=\sigma_{\infty}-\frac{\gamma\left(\rho_{P}-\rho_{Q}\right)\left(\rho_{P}^{2}+\Lambda-\alpha_{1}^{2}\right)\left(\rho_{P}^{2}+\Lambda^{\prime}-\alpha_{1}^{2}\right)}{k \sqrt{\left(\rho_{P}^{2}-h_{3}^{2}\right)} \sqrt{\left(\rho_{P}^{2}-h_{2}^{2}\right)}} I_{0}^{1}\left(\rho_{Q}\right),
$$

then equations (28) and (32) ensure that at all points on the quiescent interface $\rho=\rho_{Q}$ the nutrient concentration has, approximately, the threshold value $\sigma_{1}$. See the remark below. From (32) and (33) we deduce that

$$
\frac{I_{0}^{1}\left(\rho_{N}\right)}{\sigma_{\infty}-\sigma_{2}}=\frac{I_{0}^{1}\left(\rho_{Q}\right)}{\sigma_{\infty}-\sigma_{1}}
$$

which provides a connection between the boundaries $\rho_{N}$ and $\rho_{Q}$. Finally we note that the relations (32) and (34) together connect all three boundaries of the tumour model.

REMARK. The deviations imposed by the last two terms of (28) on the value $\sigma_{1}$ of $\sigma\left(\mathbf{r}_{Q}\right)$ are negligibly small. To see this write (28) in the symbolic form

$$
\sigma_{L}\left(\mathbf{r}_{Q}\right)=\sigma_{1}+A\left(\rho, \rho_{P}, \rho_{N}\right) S_{2}^{1}(\mu, \nu)+B\left(\rho, \rho_{P}, \rho_{N}\right) S_{2}^{2}(\mu, \nu) .
$$

Then using the values given in Tables 1 and 2 for the various parameters it can be shown that

$$
A\left(\rho, \rho_{P}, \rho_{N}\right) S_{2}^{1}(\mu, \nu)+B\left(\rho, \rho_{P}, \rho_{N}\right) S_{2}^{2}(\mu, \nu) \leq 10^{-8} \sigma_{1} .
$$

A similar argument may be applied to equation (29). That is, the error on setting $\sigma\left(\mathbf{r}_{N}\right)=\sigma_{2}$ is of the form

$$
\tilde{A}\left(\rho, \rho_{P}, \rho_{N}\right) S_{2}^{1}(\mu, \nu)+\tilde{B}\left(\rho, \rho_{P}, \rho_{N}\right) S_{2}^{2}(\mu, \nu) \leq 10^{-6} \sigma_{2} .
$$

The above analysis completes the discussion of the nutrient concentration within the tumour. We now consider the corresponding pressure distribution.

2.2. The pressure concentration problem. Combining equation (12) and (15) we find that on $\rho=\rho_{p}$,

$$
\left[h_{\rho}\left(\rho_{p}\right)\right]^{2} \frac{d \rho_{p}}{d t}=-\frac{\partial p}{\partial \rho}\left(\rho_{p}\right)+\frac{\beta}{d_{t}} h_{\rho}\left(\rho_{p}\right) s\left(\rho_{p}\right) .
$$

We now make the important observation that because of the form of the scale factor $h_{\rho}\left(\rho_{p}\right)$ and the orthogonality of the surface ellipsoidal harmonics, the right-hand side of (35) must be expressible as an expansion in terms of the surface harmonics (27).

Turning to the boundary condition (14) for the nutrient concentration $\sigma_{L}\left(\mathbf{r}_{P}\right)$ we have

$$
\frac{\partial \sigma_{L}\left(\mathbf{r}_{P}\right)}{\partial \rho}=\frac{\gamma}{k} h_{\rho}\left(\mathbf{r}_{P}\right) s\left(\mathbf{r}_{P}\right) .
$$


From (23) we see that $\sigma_{L}\left(\mathbf{r}_{P}\right)$ is expressible as an expansion in terms of the ellipsoidal harmonics (26).

On combining equations (13) and (15) we obtain the relation

$$
-h_{\mu}\left(\mathbf{r}_{P}\right) \frac{d \mu}{d t} \hat{\boldsymbol{\nu}}+h_{\nu}\left(\mathbf{r}_{P}\right) \frac{d \nu}{d t} \hat{\boldsymbol{\mu}}=-\frac{1}{h_{\mu}\left(\mathbf{r}_{P}\right)} \frac{\partial p_{L}}{\partial \mu} \hat{\boldsymbol{\nu}}+\frac{1}{h_{\nu}\left(\mathbf{r}_{P}\right)} \frac{\partial p_{L}}{\partial \nu} \hat{\boldsymbol{\mu}},
$$

from which we deduce that

$$
\begin{aligned}
{\left[h_{\mu}\left(\mathbf{r}_{P}\right)\right]^{2} \frac{d \mu}{d t} } & =\frac{\partial p_{L}}{\partial \mu} \\
{\left[h_{\nu}\left(\mathbf{r}_{P}\right)\right]^{2} \frac{d \nu}{d t} } & =\frac{\partial p_{L}}{\partial \nu} .
\end{aligned}
$$

These relations determine the evolution of cells on the outer surface of the tumour and so for consistency we must have

and consequently

$$
\frac{d \mu}{d t}=0=\frac{d \nu}{d t}
$$

$$
\frac{\partial p_{L}}{\partial \mu}=0=\frac{\partial p_{L}}{\partial \nu}
$$

On taking account of these relations, equation (9), together with (11), the boundary condition (12) and the Young-Laplace equation (17), is solved in the following manner.

In both the live cell layer and the necrotic core, equation (9) has the particular integral

$$
p_{\text {part }, i}=\frac{S_{i} \rho^{2}}{6}\left(S_{0}^{1}(\mu, \nu)-\frac{S_{2}^{1}(\mu, \nu)}{\left(\Lambda-\Lambda^{\prime}\right)\left(\Lambda-\alpha_{1}^{2}\right)}+\frac{S_{2}^{2}(\mu, \nu)}{\left(\Lambda-\Lambda^{\prime}\right)\left(\Lambda^{\prime}-\alpha_{1}^{2}\right)}\right)
$$

for $i=N, L$.

The full pressure field in the necrotic core can then be written in the form

$$
\begin{aligned}
p_{N}(\rho, \mu, \nu)= & \sum_{n=0}^{\infty} \sum_{m=1}^{2 n+1} d_{n}^{m} E_{n}^{m}(\rho) E_{n}^{m}(\mu) E_{n}^{m}(\nu) \\
& +\frac{S_{N} \rho^{2}}{6}\left(S_{0}^{1}(\mu, \nu)-\frac{S_{2}^{1}(\mu, \nu)}{\left(\Lambda-\Lambda^{\prime}\right)\left(\Lambda-\alpha_{1}^{2}\right)}+\frac{S_{2}^{2}(\mu, \nu)}{\left(\Lambda-\Lambda^{\prime}\right)\left(\Lambda^{\prime}-\alpha_{1}^{2}\right)}\right),
\end{aligned}
$$

while in the live cell layer we write

$$
\begin{aligned}
p_{L}(\rho, \mu, \nu)= & \sum_{n=0}^{\infty} \sum_{m=1}^{2 n+1}\left[e_{n}^{m}+(2 n+1) I_{n}^{m}(\rho) f_{n}^{m}\right] E_{n}^{m}(\rho) E_{n}^{m}(\mu) E_{n}^{m}(\nu) \\
& +\frac{S_{L} \rho^{2}}{6}\left(S_{0}^{1}(\mu, \nu)-\frac{S_{2}^{1}(\mu, \nu)}{\left(\Lambda-\Lambda^{\prime}\right)\left(\Lambda-\alpha_{1}^{2}\right)}+\frac{S_{2}^{2}(\mu, \nu)}{\left(\Lambda-\Lambda^{\prime}\right)\left(\Lambda^{\prime}-\alpha_{1}^{2}\right)}\right) .
\end{aligned}
$$

The coefficients $d_{n}^{m}, e_{n}^{m}, f_{n}^{m}$ are determined by applying the continuity boundary conditions together with (17), with the assumption that the trace of the exterior pressure $p_{\text {out }}$ on the outer boundary of the tumour is given by

$$
p_{\text {out }}=g\left(\rho_{P}\right)-a \kappa\left(\mathbf{r}_{P}\right) \text {. }
$$


In (43), $g\left(\rho_{P}\right)$ is a function of $\rho$, constant on the boundary $\rho=\rho_{P}$ and $\kappa\left(\mathbf{r}_{P}\right)$ is the mean curvature at the point $\mathbf{r}_{P}$ on the ellipsoid defined by

$$
\kappa\left(\rho_{p}, \mu, \nu\right)=\left(\frac{1}{\rho_{p}^{2}-\mu^{2}}+\frac{1}{\rho_{p}^{2}-\nu^{2}}\right) \frac{\rho_{p} \sqrt{\rho_{p}^{2}-h_{3}^{2}} \sqrt{\rho_{p}^{2}-h_{2}^{2}}}{2 \sqrt{\rho_{p}^{2}-\mu^{2}} \sqrt{\rho_{p}^{2}-\nu^{2}}} .
$$

Hence we arrive, for $\mathbf{r} \in \Omega_{L}$, at

$$
\begin{aligned}
p_{L}= & {\left[\frac{1}{6} S_{1}\left(\rho^{2}-\rho_{P}^{2}\right)+\frac{1}{4 \pi}\left(S_{1}-S_{2}\right) V\left(\rho_{N}\right) I_{0}^{1}\left(\rho, \rho_{P}\right)+g\left(\rho_{p}\right)\right] S_{0}^{1}(\mu, \nu) } \\
+ & \frac{1}{\left(\Lambda^{\prime}-\Lambda\right)}\left\{\frac{S_{1}}{6\left(\Lambda-a_{1}^{2}\right)}\left[\rho^{2}-\rho_{p}^{2} \frac{E_{2}^{1}(\rho)}{E_{2}^{1}\left(\rho_{p}\right)}\right]\right. \\
& \left.-\frac{1}{4 \pi}\left(S_{1}-S_{2}\right) V\left(\rho_{N}\right) I_{2}^{1}\left(\rho, \rho_{P}\right) E_{2}^{1}(\rho)\right\} S_{2}^{1}(\mu, \nu) \\
+ & \frac{1}{\left(\Lambda-\Lambda^{\prime}\right)}\left\{\frac{S_{1}}{6\left(\Lambda^{\prime}-a_{1}^{2}\right)}\left[\rho^{2}-\rho_{p}^{2} \frac{E_{2}^{2}(\rho)}{E_{2}^{2}\left(\rho_{p}\right)}\right]\right. \\
& \left.+\frac{1}{4 \pi}\left(S_{1}-S_{2}\right) V\left(\rho_{N}\right) I_{2}^{2}\left(\rho, \rho_{p}\right) E_{2}^{2}(\rho)\right\} S_{2}^{2}(\mu, \nu) .
\end{aligned}
$$

Similarly for $\mathbf{r} \in \Omega_{N}$,

$$
\begin{aligned}
p_{N}= & {\left[\frac{1}{6} S_{2}\left(\rho^{2}-\rho_{N}^{2}\right)+\frac{1}{4 \pi}\left(S_{1}-S_{2}\right) V\left(\rho_{N}\right) I_{0}^{1}\left(\rho_{N}, \rho_{P}\right)+\frac{1}{6} S_{1}\left(\rho_{N}^{2}-\rho_{P}^{2}\right)+g\left(\rho_{P}\right)\right] } \\
& +\frac{1}{\left(\Lambda-\Lambda^{\prime}\right)}\left\{\frac{S_{2}}{6\left(\Lambda-a_{1}^{2}\right)}\left[\frac{E_{2}^{1}(\rho) \rho_{N}^{2}}{E_{2}^{1}\left(\rho_{N}\right)}-\rho^{2}\right]\right. \\
& \left.\quad-\frac{S_{1}}{6\left(\Lambda-a_{1}^{2}\right)}\left[\frac{\rho_{N}^{2}}{E_{2}^{1}\left(\rho_{N}\right)}-\frac{\rho_{P}^{2}}{E_{2}^{1}\left(\rho_{P}\right)}\right] E_{2}^{1}(\rho)\right\} S_{2}^{1}(\mu, \nu) \\
& +\frac{1}{\left(\Lambda-\Lambda^{\prime}\right)}\left\{\frac{1}{4 \pi}\left(S_{1}-S_{2}\right) V\left(\rho_{N}\right) I_{2}^{1}\left(\rho_{P}, \rho_{N}\right) E_{2}^{1}(\rho)\right\} S_{2}^{1}(\mu, \nu) \\
& \frac{1}{\left(\Lambda-\Lambda^{\prime}\right)}\left\{\frac{S_{2}}{6\left(\Lambda^{\prime}-a_{1}^{2}\right)}\left[\frac{E_{2}^{1}(\rho) \rho_{N}^{2}}{E_{2}^{1}\left(\rho_{N}\right)}-\rho^{2}\right]\right. \\
& \left.-\frac{1}{6\left(\Lambda^{\prime}-a_{1}^{2}\right)}\left[\frac{\rho_{N}^{2}}{E_{2}^{2}\left(\rho_{N}\right)}-\frac{\rho_{P}^{2}}{E_{2}^{2}\left(\rho_{P}\right)}\right] E_{2}^{2}(\rho)\right\} S_{2}^{2}(\mu, \nu) \\
& \frac{1}{\left(\Lambda-\Lambda^{\prime}\right)}\left\{\frac{1}{4 \pi}\left(S_{1}-S_{2}\right) V\left(\rho_{N}\right) I_{2}^{2}\left(\rho_{P}, \rho_{N}\right) E_{2}^{2}(\rho)\right\} S_{2}^{2}(\mu, \nu),
\end{aligned}
$$

where $I_{n}^{m}\left(\rho_{1}, \rho_{2}\right)=I_{n}^{m}\left(\rho_{1}\right)-I_{n}^{m}\left(\rho_{2}\right)$. 
Using the definitions of the Lamé polynomials (26), the expressions (44) and (45) can be written as

$$
\begin{aligned}
p_{L} & =\left[\frac{1}{6} S_{1}\left(\rho^{2}-\rho_{P}^{2}\right)+\frac{1}{4 \pi}\left(S_{1}-S_{2}\right) V\left(\rho_{N}\right) I_{0}^{1}\left(\rho, \rho_{P}\right)+g\left(\rho_{P}\right)\right] \\
& +\left\{\frac{\left(S_{1}-S_{2}\right) V\left(\rho_{N}\right)}{4 \pi\left(\Lambda-\Lambda^{\prime}\right)} I_{2}^{1}\left(\rho_{P}, \rho\right)\left(\rho^{2}+\Lambda-a_{1}^{2}\right)+\frac{S_{1}\left(\rho_{P}^{2}-\rho^{2}\right)}{6\left(\Lambda-\Lambda^{\prime}\right)\left(\rho_{P}^{2}+\Lambda-a_{1}^{2}\right)}\right\} S_{2}^{1}(\mu, \nu) \\
& -\left\{\frac{\left(S_{1}-S_{2}\right) V\left(\rho_{N}\right)}{4 \pi\left(\Lambda-\Lambda^{\prime}\right)} I_{2}^{2}\left(\rho_{P}, \rho\right)\left(\rho^{2}+\Lambda^{\prime}-a_{1}^{2}\right)+\frac{S_{1}\left(\rho_{P}^{2}-\rho^{2}\right)}{6\left(\Lambda-\Lambda^{\prime}\right)\left(\rho_{P}^{2}+\Lambda^{\prime}-a_{1}^{2}\right)}\right\} S_{2}^{2}(\mu, \nu)
\end{aligned}
$$

and

$$
\begin{aligned}
p_{N} & =\left[\frac{1}{6} S_{2}\left(\rho^{2}-\rho_{N}^{2}\right)+\frac{1}{4 \pi}\left(S_{1}-S_{2}\right) V\left(\rho_{N}\right) I_{0}^{1}\left(\rho_{N}, \rho_{P}\right)+\frac{1}{6} S_{1}\left(\rho_{N}^{2}-\rho_{P}^{2}\right)+g\left(\rho_{P}\right)\right] \\
& +\left\{\frac{\left(S_{1}-S_{2}\right) V\left(\rho_{N}\right)}{4 \pi\left(\Lambda-\Lambda^{\prime}\right)} I_{2}^{1}\left(\rho_{P}, \rho_{N}\right)\left(\rho^{2}+\Lambda-a_{1}^{2}\right)\right. \\
& \left.+\frac{S_{1}\left(\rho_{P}^{2}-\rho_{N}^{2}\right)\left(\rho^{2}+\Lambda-a_{1}^{2}\right)}{6\left(\Lambda-\Lambda^{\prime}\right)\left(\rho_{P}^{2}+\Lambda-a_{1}^{2}\right)\left(\rho_{N}^{2}+\Lambda-a_{1}^{2}\right)}\right\} S_{2}^{1}(\mu, \nu) \\
& +\left\{\frac{S_{2}\left(\rho_{N}^{2}-\rho^{2}\right)}{6\left(\Lambda-\Lambda^{\prime}\right)\left(\rho_{N}^{2}+\Lambda-a_{1}^{2}\right)}\right\} S_{2}^{1}(\mu, \nu) \\
& -\left\{\frac{\left(S_{1}-S_{2}\right) V\left(\rho_{N}\right)}{4 \pi\left(\Lambda-\Lambda^{\prime}\right)} I_{2}^{2}\left(\rho_{P}, \rho_{N}\right)\left(\rho^{2}+\Lambda^{\prime}-a_{1}^{2}\right)\right. \\
& \left.+\frac{S_{1}\left(\rho_{P}^{2}-\rho_{N}^{2}\right)\left(\rho^{2}+\Lambda^{\prime}-a_{1}^{2}\right)}{6\left(\Lambda-\Lambda^{\prime}\right)\left(\rho_{P}^{2}+\Lambda^{\prime}-a_{1}^{2}\right)\left(\rho_{N}^{2}+\Lambda^{\prime}-a_{1}^{2}\right)}\right\} S_{2}^{2}(\mu, \nu) \\
& -\left\{\frac{S_{2}\left(\rho_{N}^{2}-\rho^{2}\right)}{6\left(\Lambda-\Lambda^{\prime}\right)\left(\rho_{N}^{2}+\Lambda^{\prime}-a_{1}^{2}\right)}\right\} S_{2}^{2}(\mu, \nu) .
\end{aligned}
$$

The next problem is to derive the differential equation that describes the evolution of the tumour's outer boundary. On noting that the thickness of the layer of live cells is

$$
s=h_{\rho}\left(\rho_{p}\right)\left(\rho_{P}-\rho_{Q}\right)
$$

we find, from (35), that

$$
\left[h_{\rho}\left(\mathbf{r}_{P}\right)\right]^{2} \frac{d \rho_{p}}{d t}=-\frac{\partial p_{L}}{\partial \rho}\left(\mathbf{r}_{P}\right)+\frac{\beta}{d_{t}}\left[h_{\rho}\left(\mathbf{r}_{P}\right)\right]^{2}\left(\rho_{P}-\rho_{Q}\right) .
$$

On substituting for $p_{L}$ from (47) in (50) and using the orthogonality property of the surface ellipsoidal harmonics on $\rho=\rho_{P}$ we find

$$
\begin{aligned}
\frac{d \rho_{P}}{d t}= & \frac{\left(S_{1}-S_{2}\right) V\left(\rho_{N}\right)}{4 \pi} \frac{\sqrt{\left(\rho_{P}^{2}-h_{3}^{2}\right)\left(\rho_{P}^{2}-h_{2}^{2}\right)}}{\left(\rho_{P}^{2}+\Lambda-\alpha_{1}^{2}\right)\left(\rho_{P}^{2}+\Lambda^{\prime}-\alpha_{1}^{2}\right)} \\
& -\frac{S_{1}}{3} \frac{\rho_{P}\left(\rho_{P}^{2}-h_{3}^{2}\right)\left(\rho_{P}^{2}-h_{2}^{2}\right)}{\left(\rho_{P}^{2}+\Lambda-\alpha_{1}^{2}\right)\left(\rho_{P}^{2}+\Lambda^{\prime}-\alpha_{1}^{2}\right)}+\frac{\beta}{d_{t}}\left(\rho_{P}-\rho_{Q}\right)
\end{aligned}
$$


or

$\frac{d \rho_{P}}{d t}=-\left[\frac{S_{2} V\left(\rho_{N}\right)}{V\left(\rho_{P}\right)}+\frac{S_{1}\left(V\left(\rho_{P}\right)-V\left(\rho_{N}\right)\right)}{V\left(\rho_{P}\right)}\right] \frac{\rho_{P}\left(\rho_{P}^{2}-h_{3}^{2}\right)\left(\rho_{P}^{2}-h_{2}^{2}\right)}{3\left(\rho_{P}^{2}+\Lambda-\alpha_{1}^{2}\right)\left(\rho_{P}^{2}+\Lambda^{\prime}-\alpha_{1}^{2}\right)}+\frac{\beta}{d_{t}}\left(\rho_{P}-\rho_{Q}\right)$,

where $V\left(\rho_{P}\right), V\left(\rho_{N}\right)$ are the volumes of the ellipsoids $\rho=\rho_{P}$ and $\rho=\rho_{N}$, respectively, given by equation (30).

3. The spherical tumour. In this section we revisit the problem of a tumour constrained to grow as a sphere. In particular we are concerned with the model introduced in this paper in which the nutrient $\sigma$ satisfies the boundary condition

$$
\hat{\mathbf{n}} \cdot \nabla \sigma=\frac{\gamma}{k} s
$$

where $s$ is the depth of the outer layer of live proliferating cells. This contrasts with the Greenspan model in which $\sigma$ is assumed to satisfy the boundary condition

$$
\hat{\mathbf{n}} \cdot \nabla \sigma=\frac{\lambda}{\beta} \sqrt{\sigma(\mathbf{r})-\sigma_{1}}
$$

In analogy with the segmentation of the ellipsoidal tumour we define

$$
\begin{aligned}
& \Omega_{N}=\left\{(r, \theta, \phi): 0 \leq r<r_{N}, \sigma(\mathbf{r})=\sigma_{2}\right\}, \\
& \Omega_{Q}=\left\{(r, \theta, \phi): r_{N} \leq r<r_{Q}, \sigma_{2}<\sigma(\mathbf{r})<\sigma_{1}\right\}, \\
& \Omega_{P}=\left\{(r, \theta, \phi): r_{Q} \leq r<r_{P}, \sigma(\mathbf{r})>\sigma_{1}\right\}, \\
& \Omega_{S}=\left\{(r, \theta, \phi): r>r_{P}, \sigma_{1}<\sigma(\mathbf{r})<\sigma_{\infty}\right\} .
\end{aligned}
$$

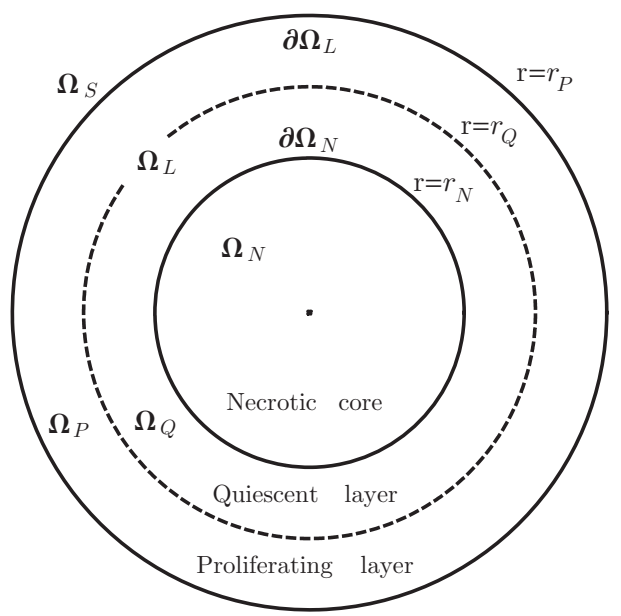

FIG. 2. Spherical tumour with the 3-layer structure of necrotic core, quiescent layer, and proliferating layer.

In analogy with the analysis for the ellipsoidal tumour we find that the nutrient concentration in the proliferating and quiescent layers is given by

$$
\sigma(\mathbf{r})=\sigma_{\infty}-\frac{\gamma}{k} \frac{r_{P}^{2}\left(r_{P}-r_{Q}\right)}{r}, \mathbf{r} \in \Omega_{P} \cup \Omega_{Q}
$$


which holds as long as $\sigma>\sigma_{2}$.

In the necrotic core we deduce that

$$
\sigma(\mathbf{r})=\sigma_{2}, \mathbf{r} \in \Omega_{N} .
$$

From (55) we can determine a relationship between the radius $r_{P}$ of the tumour and the radius $r_{Q}$ of the quiescent layer by observing that when $\sigma=\sigma_{1}$,

$$
\sigma\left(r_{Q}\right)=\sigma_{1}=\sigma_{\infty}-\frac{\gamma}{k} \frac{r_{p}^{2}\left(r_{P}-r_{Q}\right)}{r_{Q}}, r_{Q} \in \Omega_{P} \cup \Omega_{Q},
$$

from which we find that

$$
r_{Q}=\frac{\gamma r_{P}^{3}}{k\left(\sigma_{\infty}-\sigma_{1}\right)+\gamma r_{P}^{2}} .
$$

Similarly on $\sigma=\sigma_{2}$ we have the continuity condition

$$
\sigma\left(r_{N}\right)=\sigma_{2}=\sigma_{\infty}-\frac{\gamma}{k} \frac{r_{P}^{2}\left(r_{P}-r_{Q}\right)}{r_{N}}, r_{N} \in \partial \Omega_{N}
$$

from which we deduce that

$$
r_{N}=\left(\frac{\sigma_{\infty}-\sigma_{1}}{\sigma_{\infty}-\sigma_{2}}\right) r_{Q}
$$

Now consider the determination of the pressure field $p(\mathbf{r})$ in the interior and exterior of spherical tumour. As in section 2, the pressure satisfies the partial differential equation (9) together with the boundary condition (17) and the continuity of pressure and its normal derivative across each internal layer. As in section 2 we assume that the tumour continues to grow as a sphere and that on its external boundary

$$
p_{\text {out }}\left(\mathbf{r}_{\mathbf{P}}\right)=g\left(r_{P}\right)-\frac{\alpha}{r_{P}} .
$$

Omitting the details of the calculations, which mimic those of section 2 , we find that the pressure field in the necrotic core is given by

$$
p_{N}(\mathbf{r})=g\left(r_{P}\right)+\frac{1}{6}\left(S_{2} r^{2}-S_{1} r_{P}^{2}\right)+\left(S_{1}-S_{2}\right) r_{N}^{3}\left[\frac{1}{2 r_{N}}-\frac{1}{3 r_{P}}\right], \mathbf{r} \in \Omega_{N},
$$

and

$$
p_{L}(\mathbf{r})=g\left(r_{P}\right)+\frac{1}{6} S_{1}\left(r_{P}^{2}-r^{2}\right)+\frac{1}{3}\left(S_{1}-S_{2}\right) r_{N}^{3}\left[\frac{1}{r}-\frac{1}{r_{P}}\right], \mathbf{r} \in \Omega_{Q} \cup \Omega_{P} .
$$

In order to derive an equation governing the evolution of the outer tumour boundary we turn to equation (12), which in spherical polar coordinates becomes

$$
\frac{d r_{P}}{d t}=\frac{d p_{L}(\mathbf{r})}{d r}+\frac{\beta}{d_{t}}\left(r_{P}-r_{Q}\right), \mathbf{r} \in \partial \Omega_{P} .
$$

By substituting (61) into (62) we obtain the following nonlinear ordinary differential equation

$$
\frac{d r_{P}}{d t}=\frac{\left(S_{1}-S_{2}\right) r_{N}^{3}}{3 r_{P}^{2}}-\frac{S_{1} r_{P}}{3}+\frac{\beta}{d_{t}}\left(r_{P}-r_{Q}\right) .
$$

On substituting for $r_{Q}$ and $r_{N}$ from (57) and (58) in (63) we obtain

$$
\frac{d r_{P}}{d t}=\frac{1}{3}\left(\frac{\sigma_{\infty}-\sigma_{1}}{\sigma_{\infty}-\sigma_{2}}\right)^{3} \frac{\left(S_{1}-S_{2}\right) r_{P}^{7}}{\left(k / \gamma\left(\sigma_{\infty}-\sigma_{1}\right)+r_{P}^{2}\right)^{3}}-\frac{1}{3} S_{1} r_{P}+\frac{\beta}{d_{t}} \frac{k\left(\sigma_{\infty}-\sigma_{1}\right) r_{P}}{k\left(\sigma_{\infty}-\sigma_{1}\right)+\gamma r_{P}^{2}}
$$


TABLE 1

\begin{tabular}{|l|r|c|}
\hline Tumour type & Initial tumour dimensions & Initial Tumour Volume \\
\hline Sphere & $a=6 / 1000$ & \\
\hline Ellipsoid I & $a_{1}=7.2 / 1000, a_{2}=6 / 1000, a_{3}=5 / 1000$ & $\frac{4 \pi}{3}(6 / 1000)^{3}$ \\
\hline Ellipsoid II & $a_{1}=9 / 1000, a_{2}=6 / 1000, a_{3}=4 / 1000$ & \\
\hline
\end{tabular}

TABLE 2

Set of Parameters

\begin{tabular}{|c|r|c|}
\hline $1 \mathrm{a}$ & $\sigma_{1}=0.9, \sigma_{2}=0.4, \gamma / k=5000$ & $\beta / d_{t}=500$ \\
\hline $1 \mathrm{~b}$ & $\sigma_{1}=0.9, \sigma_{2}=0.4, \gamma / k=5000$ & $\beta / d_{t}=1000$ \\
\hline $\mathrm{2a}$ & $\sigma_{1}=0.9, \sigma_{2}=0.875, \gamma / k=5000$ & $\beta / d_{t}=500$ \\
\hline $2 \mathrm{~b}$ & $\sigma_{1}=0.9, \sigma_{2}=0.875, \gamma / k=5000$ & $\beta / d_{t}=1000$ \\
\hline
\end{tabular}

To conclude this section it is appropriate to highlight the main modelling differences between the above model and that of Greenspan [6, 7. Firstly, in Greenspan's approach, the gradient of the nutrient concentration was assumed to obey a square root law on the tumour's outer boundary whereas in our model we simply assume that the gradient of the nutrient concentration is proportional to the live cell depth. As a particular consequence of this it can be shown that the difference between the tumour surface rate of growth using the Greenspan model and the surface rate of growth in the above model is $\mathcal{O}\left(\frac{1}{r_{P}}\right)$. Secondly, we assume that in the exterior of the tumour, the pressure equipotential surfaces are spheres concentric with the spherical tumour and that its trace on the tumour's outer boundary is governed by the Young-Laplace relation which is not reflected in the evolution of the tumour.

4. Numerical simulations. In this section we have carried out a series of numerical experiments which compare the evolution of different initial ellipsoidal tumours (with the same initial volume). As a reference we use a spherical tumour (with the same initial volume).

The numerical experiments examined the time evolution of the volumes of the boundaries $r=r_{N}, r=r_{Q}$ and $r=r_{P}$ (for the spherical tumour) and the volumes of the boundaries $\rho=\rho_{N}, \rho=\rho_{Q}$ and $\rho=\rho_{P}$ (for the ellipsoidal tumours). In all cases the initial tumours have the same volume but different initial dimensions, as shown in Table 1 .

The simulations have been carried out for the parameter values shown in Table 2, We present in Figures 4 and 5 only the cases of sets 1a and 2a.

For the set $1 \mathrm{a}, \sigma_{1}-\sigma_{2}=0.5$ and $\beta / d_{t}=500$ (Figure 3), all spherical boundaries exist but are relatively smaller than the corresponding ellipsoidal boundaries. Specifically, the necrotic core boundary for the eccentric ellipsoid assumes the value $\rho_{N}=h_{2}$ which corresponds to the zero volume of the two-dimensional focal ellipse. For the set 1b, $\sigma_{1}-\sigma_{2}=0.5$ and $\beta / d_{t}=1000$, the results are much the same except for the eccentric ellipsoid which maintains the two-dimensional focal ellipse as its necrotic core. 


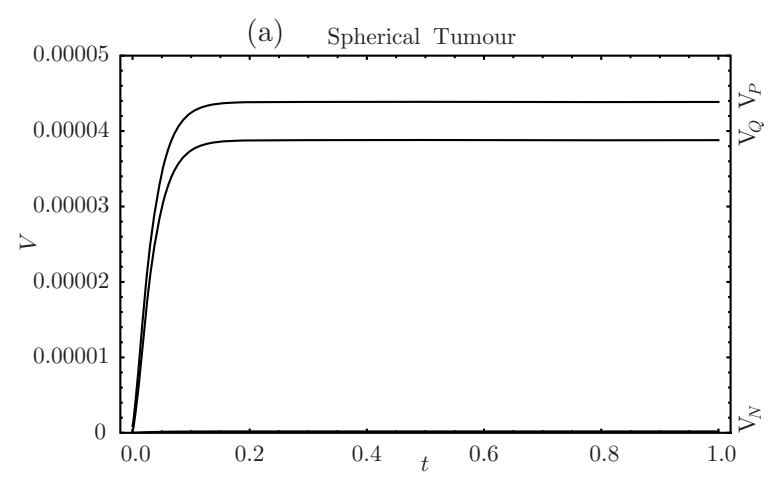

(b) Ellipsoidal Tumour I

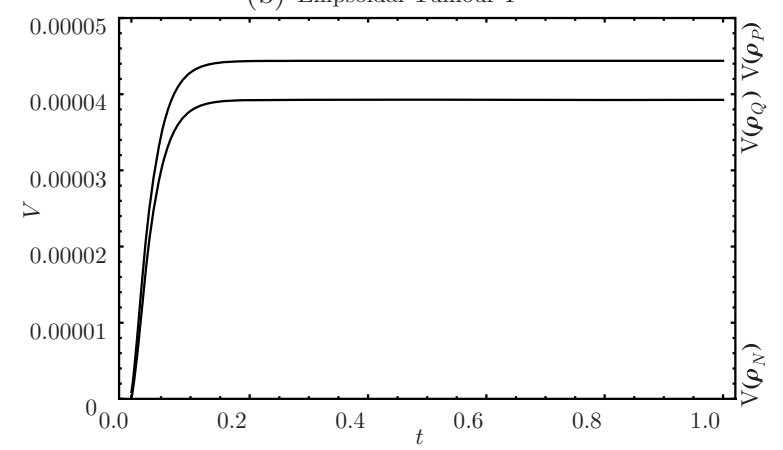

(c) Ellipsoidal Tumour II

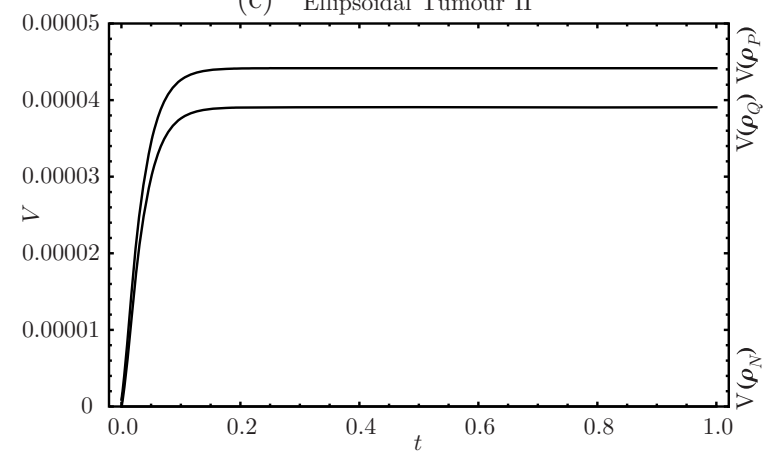

FIG. 3. Volume time evolution of the boundaries (a) $r=r_{N}, r=$ $r_{Q}$ and $r=r_{P}$ for a spherical tumor with initial radii $6 / 1000$, (b) $\rho=\rho_{N}, \rho=\rho_{Q}$ and $\rho=\rho_{P}$ for an ellipsoidal tumor with initial dimensions $a_{1}=7.2 / 1000, a_{2}=6 / 1000, a_{3}=5 / 1000$, (c) $\rho=\rho_{N}$, $\rho=\rho_{Q}$ and $\rho=\rho_{P}$ for an ellipsoidal tumor with initial dimensions $a_{1}=7.2 / 1000, a_{2}=6 / 1000, a_{3}=5 / 1000$, for the set of parameters $\sigma_{1}=0.9, \sigma_{2}=0.4, \gamma / k=5000$ and $\beta / d_{t}=500$.

For the set $2 \mathrm{a}$ we take $\sigma_{1}-\sigma_{2}=0.025$ and $\beta / d_{t}=500$ (Figure 4) where all the boundaries are larger. The new observation is that for the eccentric ellipsoid a definite necrotic core appears which is nevertheless still smaller than that for the sphere or the quasi-spherical tumour. For the set $2 \mathrm{~b}$ we take $\sigma_{1}-\sigma_{2}=0.025$ and $\beta / d_{t}=1000$; 
(a) Spherical Tumour
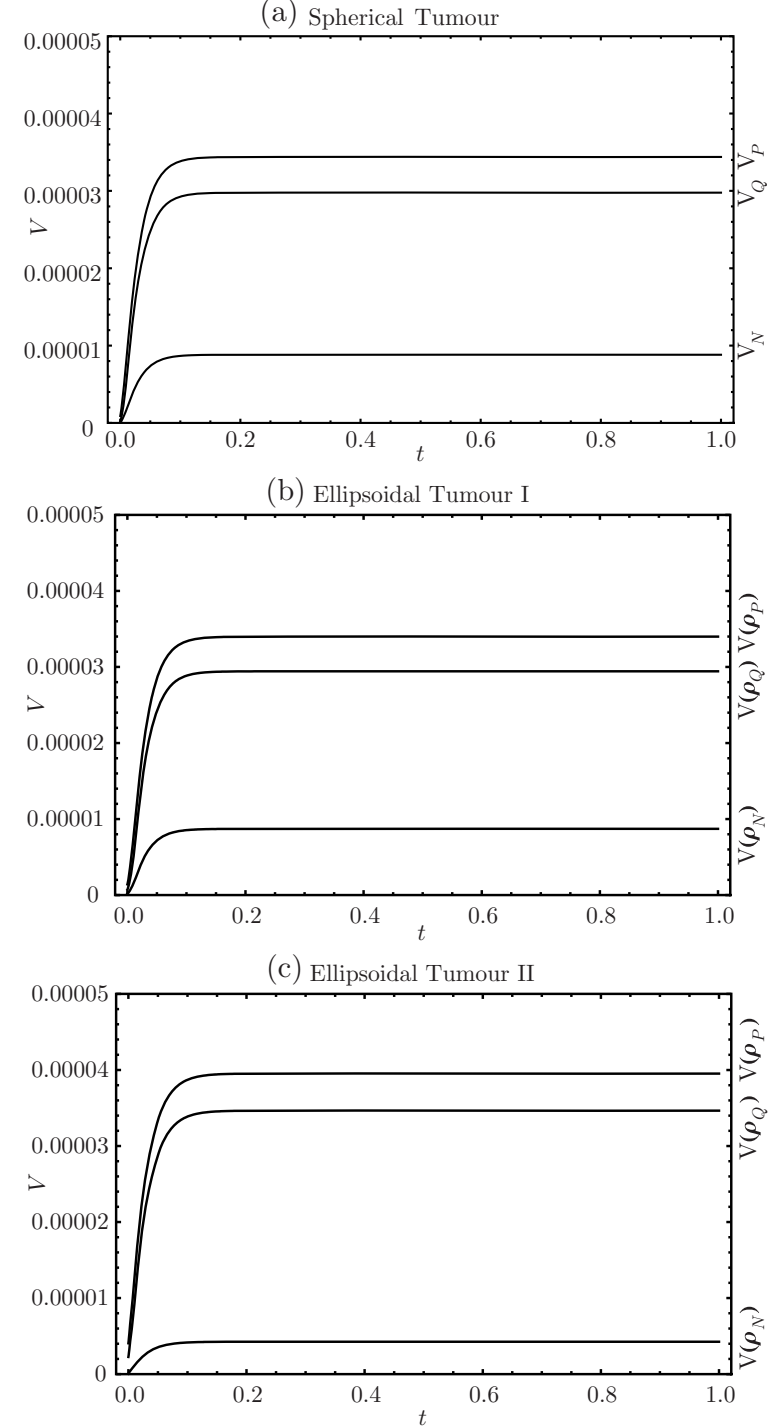

FIG 4. Volume time evolution of the boundaries (a) $r=r_{N}, r=r_{Q}$ and $r=r_{P}$ for a spherical tumor with initial radii $6 / 1000,(\mathrm{~b}) \rho=\rho_{N}$, $\rho=\rho_{Q}$ and $\rho=\rho_{P}$ for an ellipsoidal tumor with initial dimensions $a_{1}=7.2 / 1000, a_{2}=6 / 1000, a_{3}=5 / 1000$, (c) $\rho=\rho_{N}, \rho=\rho_{Q}$ and $\rho=\rho_{P}$ for an ellipsoidal tumor with initial dimensions $a_{1}=$ $7.2 / 1000, a_{2}=6 / 1000, a_{3}=5 / 1000$, for the set of parameters $\sigma_{1}=$ $0.9, \sigma_{2}=0.875, \gamma / k=5000$ and $\beta / d_{t}=500$ (set $2 \mathrm{a}$ ).

here, with a much greater proliferation parameter, there is little qualitative difference as compared with set $2 \mathrm{a}$ except that the resulting tumours are somewhat larger.

From these simulations the difference $\sigma_{1}-\sigma_{2}$ appears to play a critical role in the development of the necrotic core of a growing ellipsoidal tumour. That is, for the larger 
difference $\sigma_{1}-\sigma_{2}=0.5$, the necrotic core appears to be confined to the two-dimensional focal ellipse whereas for the smaller difference $\sigma_{1}-\sigma_{2}=0.025$, the necrotic core develops as an ellipsoid. This is a departure from the growth of a spherical tumour where the necrotic core is always a sphere.

To explore this a little further we have plotted (Figure 5), for spherical tumours, the functions

$$
f_{N, s}=\frac{1}{r_{N}\left(\sigma_{\infty}-\sigma_{2}\right)}
$$

and

$$
f_{Q, s}=\frac{1}{r_{Q}\left(\sigma_{\infty}-\sigma_{1}\right)},
$$

for a range of values of $r_{N}$ and $r_{Q}$ from which we see that (58) holds for nonzero values of $r_{N}$ and $r_{Q}$.

Similarly, for ellipsoidal tumours we make the analogous plots (Figure 5) of

$$
f_{N, e}=\frac{I_{0}^{1}\left(\rho_{N}\right)}{\left(\sigma_{\infty}-\sigma_{2}\right)}
$$

and

$$
f_{Q, e}=\frac{I_{0}^{1}\left(\rho_{N}\right)}{\left(\sigma_{\infty}-\sigma_{1}\right)}
$$

valid for $\rho \geq h_{2}$. Again we see that there is a range of values of $\rho_{N}$ and $\rho_{Q}$ for which the necessary equality

$$
\frac{I_{0}^{1}\left(\rho_{N}\right)}{\left(\sigma_{\infty}-\sigma_{2}\right)}=\frac{I_{0}^{1}\left(\rho_{Q}\right)}{\left(\sigma_{\infty}-\sigma_{1}\right)}
$$

holds.

5. Conclusions. Ellipsoidal avascular tumours have been observed to grow as a consequence of tissue stress as for example in breast cancer in women. Although a number of analytical studies have been made of spherical tumours, this is the first study of the more complex ellipsoidal tumour. By basing the modelling on the fundamental work of Greenspan we have formulated the growth of ellipsoidal tumours in an ellipsoidal coordinate geometry setting. Although the resulting differential equations can be solved by separation of variables techniques, the underlying functions are the more complex Lamé functions. It is shown that, in contrast to the spherical tumour, the necrotic core, depending on critical nutrient levels, may simply be a two-dimensional focal ellipse. This is a feature not observed in the spherical case. Unlike the case of a spherical tumour in which a linear stability analysis can be easily carried out, the ellipsoidal case is much more demanding and will be considered in future work. Since the work of Greenspan there have been substantial advances in our understanding of avascular tumour development. This has led to a number of modifications to the original models. Nevertheless the techniques used to study these later spherical models have remained essentially the same. We believe the same to be true in the case of an ellipsoidal tumour model. However, here we expect to uncover new phenomena which are not present in the constraints of spherical geometry. We shall explore these aspects in future research. 

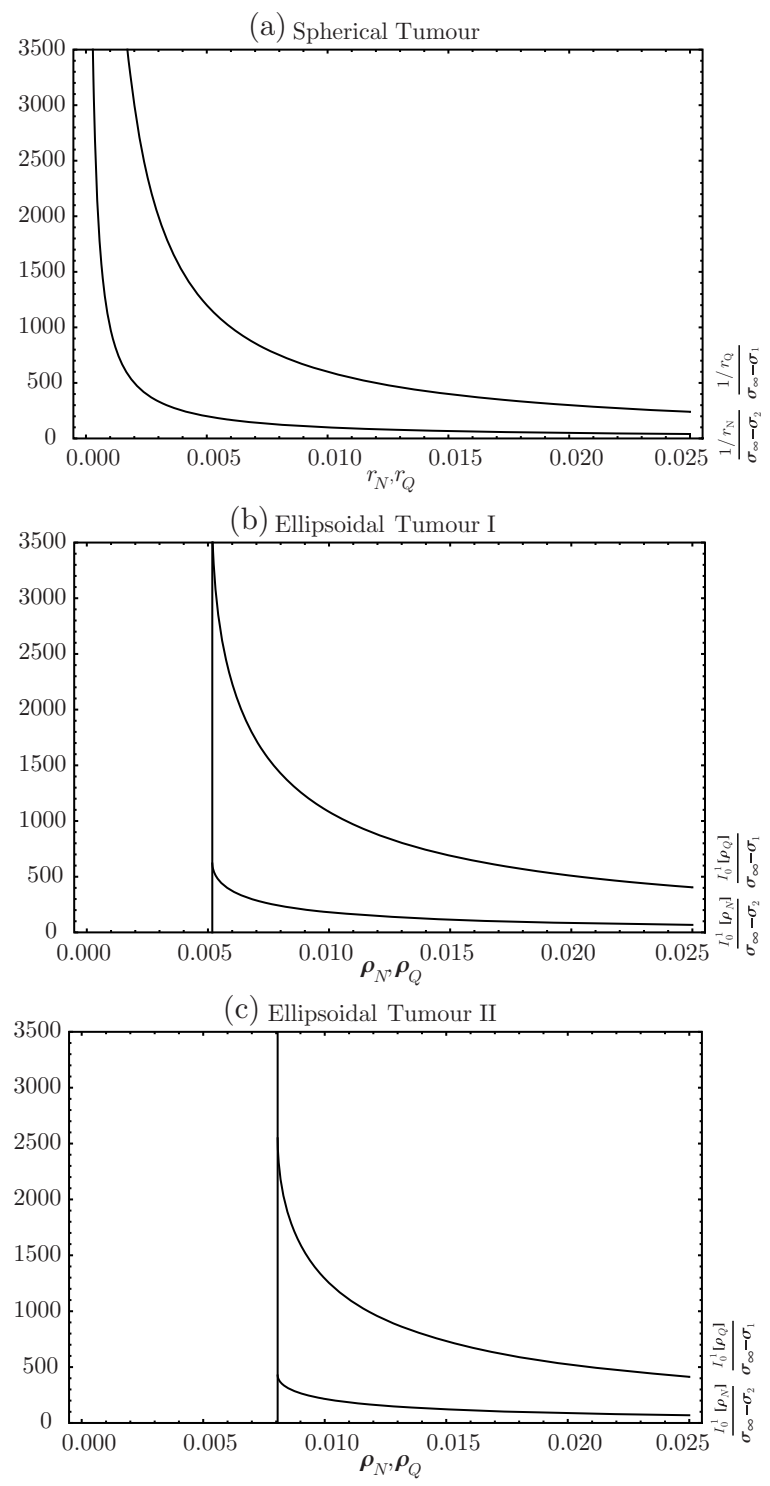

FIG. 5. Diagrams (a)-(c)

Appendix A. Ellipsoidal coordinates and Lamé products. The spherical coordinate system is completely specified if we know a centre and the radius of the unit sphere, i.e. a single unit of length. On the other hand, the ellipsoidal coordinate system, which is intrinsically three-dimensional, is specified by its centre and the three principal axes of the fundamental ellipsoid, which acts as its unit ellipsoid. Given any three numbers $\alpha_{1}, \alpha_{2}, \alpha_{3}$, with $0<\alpha_{3}<\alpha_{2}<\alpha_{1}<\infty$, we define the fundamental ellipsoid

$$
\frac{x_{1}^{2}}{\alpha_{1}^{2}}+\frac{x_{2}^{2}}{\alpha_{2}^{2}}+\frac{x_{3}^{2}}{\alpha_{3}^{2}}=1, \quad 0<\alpha_{3}<\alpha_{2}<\alpha_{1}<+\infty
$$



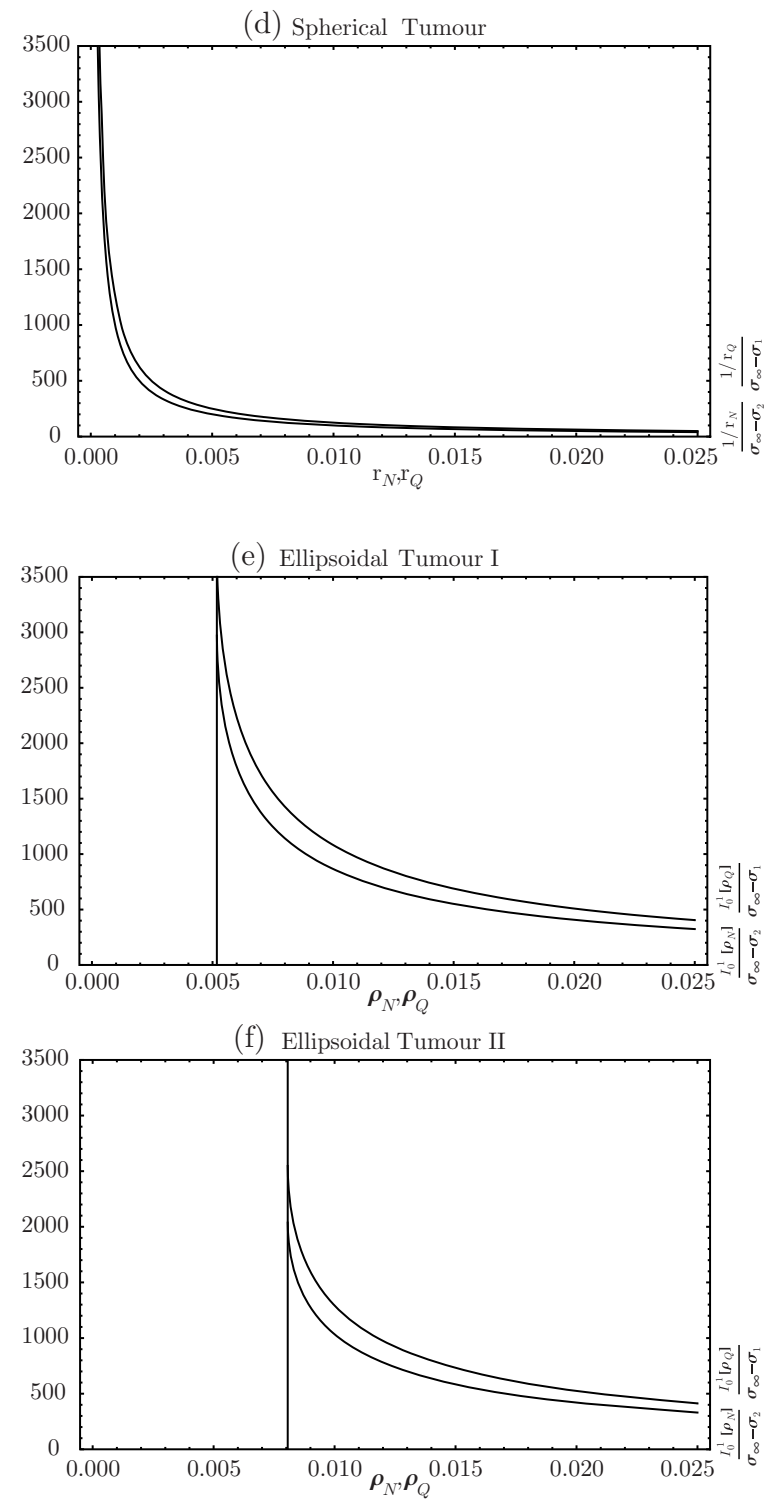

FIG. 5. Relation between the quiescent boundary and the boundary of necrotic core (a) $r=r_{Q}, r=r_{N}$, obtained by the equality $\frac{1}{r_{Q}\left(\sigma_{\infty}-\sigma_{1}\right)}=\frac{1}{r_{N}\left(\sigma_{\infty}-\sigma_{2}\right)}$, for a spherical tumor with initial radii $6 / 1000$, (b) and (c) $\rho=\rho_{Q}$ and $\rho=\rho_{N}$, obtained by the equality $\frac{I_{0}^{1}\left(\rho_{Q}\right)}{r_{Q}\left(\sigma_{\infty}-\sigma_{1}\right)}=\frac{I_{0}^{1}\left(\rho_{N}\right)}{r_{N}\left(\sigma_{\infty}-\sigma_{2}\right)}$ for an ellipsoidal tumor with initial dimensions $a_{1}=7.2 / 1000, a_{2}=6 / 1000, a_{3}=5 / 1000$ and $a_{1}=9 / 1000, a_{2}=6 / 1000, a_{3}=4 / 1000$ correspondingly, for the set of parameters $\sigma_{1}=0.9, \sigma_{2}=0.4, \gamma / k=5000$. The relation between the quiescent boundary and the boundary of the necrotic core for the set of parameters $\sigma_{1}=0.9, \sigma_{2}=0.4, \gamma / k=5000$ is represented in (d), (e) and (f). 
where the three semi-axes $\alpha_{1}, \alpha_{2}, \alpha_{3}$, specify the semi-focal distances

$$
h_{1}^{2}=\alpha_{2}^{2}-\alpha_{3}^{2}, h_{2}^{2}=\alpha_{1}^{2}-\alpha_{3}^{2}, h_{3}^{2}=\alpha_{1}^{2}-\alpha_{2}^{2},
$$

which are related by

$$
h_{1}^{2}-h_{2}^{2}+h_{3}^{2}=0 .
$$

Obviously, the foci $\pm h_{2}$ and $\pm h_{3}$ are located on the $x_{1}$-axis and the foci $\pm h_{1}$ are located on the $x_{2}$-axis. The axis $x_{3}$, hosting the shortest semi-axis, carries no foci. The centre of the ellipsoidal system is represented by the focal ellipse, i.e., an ellipse on the $\left(x_{1}, x_{2}\right)$ plane with semi-focal distance $h_{3}$ and semi-axes $h_{1}$ and $h_{2}$.

The ellipsoidal coordinate system $(\rho, \mu, \nu)$ [9], associated with the fundamental ellipsoid is connected to the Cartesian system $\left(x_{1}, x_{2}, x_{3}\right)$ by

$$
\begin{aligned}
& x_{1}=\frac{\rho \mu \nu}{h_{2} h_{3}}, \quad h_{2}<\rho<+\infty, \\
& x_{2}=\frac{\sqrt{\rho^{2}-h_{3}^{2}} \sqrt{\mu^{2}-h_{3}^{2}} \sqrt{h_{3}^{2}-\nu^{2}}}{h_{1} h_{3}}, h_{3}<\mu<h_{2}, \\
& x_{3}=\frac{\sqrt{\rho^{2}-h_{2}^{2}} \sqrt{h_{2}^{2}-\mu^{2}} \sqrt{h_{2}^{2}-\nu^{2}}}{h_{1} h_{2}}, 0<\nu<h_{3} .
\end{aligned}
$$

As depicted in Figure 6, the variable $\rho$, which corresponds to the radial spherical coordinate, defines a family of confocal ellipsoids while the variables $\mu$ and $\nu$, which correspond to the angular spherical coordinates, define two confocal families of hyperboloids of one and two sheets, respectively.

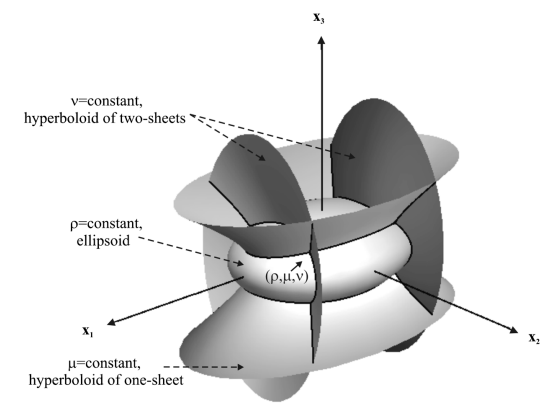

FIG. 6 Confocal ellipsoidal coordinates

The matrix coefficients of the ellipsoidal system are given by

$$
\begin{aligned}
& h_{\rho}=\left\|\mathbf{r}_{\rho}\right\|=\frac{\sqrt{\left(\rho^{2}-\mu^{2}\right)\left(\rho^{2}-\nu^{2}\right)}}{\sqrt{\left(\rho^{2}-h_{3}^{2}\right)\left(\rho^{2}-h_{2}^{2}\right)}}, \\
& h_{\mu}=\left\|\mathbf{r}_{\mu}\right\|=\frac{\sqrt{\left(\rho^{2}-\mu^{2}\right)\left(\mu^{2}-\nu^{2}\right)}}{\sqrt{\left(\mu^{2}-h_{3}^{2}\right)\left(h_{2}^{2}-\mu^{2}\right)}}, \\
& h_{\nu}=\left\|\mathbf{r}_{\nu}\right\|=\frac{\sqrt{\left(\rho^{2}-\mu^{2}\right)\left(\mu^{2}-\nu^{2}\right)}}{\sqrt{\left(h_{3}^{2}-\nu^{2}\right)\left(h_{2}^{2}-\nu^{2}\right)}},
\end{aligned}
$$


where the lower index in $\mathbf{r}$ denotes partial differentiation with respect to the indicated variable.

Lamé used an ingenious technique to separate variables for Laplace's equation. In fact, he proved that for all three separated ordinary differential equations, the only difference among them is the domain wherein each one is defined. Hence, an interior eigensolution of Laplace's equation has the form

$$
\mathbb{E}_{n}^{m}(\rho, \mu, \nu)=E_{n}^{m}(\rho) E_{n}^{m}(\mu) E_{n}^{m}(\nu),
$$

where $E_{n}^{m}$ is the Lamé function of the first kind, of degree $n=0,1,2, \ldots$, and order $m=1,2, \ldots, 2 n+1$.

Similarly, an exterior eigensolution has the form

$$
\mathbb{F}_{n}^{m}(\rho, \mu, \nu)=F_{n}^{m}(\rho) E_{n}^{m}(\mu) E_{n}^{m}(\nu),
$$

where $F_{n}^{m}$ is the Lamé function of the second kind, which is given by the elliptic integral

$$
F_{n}^{m}(\rho)=(2 n+1) E_{n}^{m}(\rho) \int_{\rho}^{\infty} \frac{d x}{\left[E_{n}^{m}(x)\right]^{2} \sqrt{\left(x^{2}-h_{3}^{2}\right)\left(x^{2}-h_{2}^{2}\right)}} .
$$

The functions $\mathbb{E}_{n}^{m}(\rho, \mu, \nu)$ and $\mathbb{F}_{n}^{m}(\rho, \mu, \nu)$ are called Lamé products or interior and exterior ellipsoidal harmonics, respectively. Note that, just as with the case of spherical geometry, there are $2 n+1$ independent ellipsoidal harmonics of degree $n$.

The surface ellipsoidal harmonics

$$
S_{n}^{m}(\mu, \nu)=E_{n}^{m}(\mu) E_{n}^{m}(\nu), n=0,1,2, \ldots, m=1,2, \ldots, 2 n+1,
$$

form a complete set of eigenfunctions over the surface $S_{\rho}$ of any ellipsoid $\rho=$ constant and satisfy the orthogonality relation

$$
\oint_{S_{\rho}} S_{n}^{m}(\mu, \nu) S_{n^{\prime}}^{m^{\prime}}(\mu, \nu) l_{\rho}(\mu, \nu) d s_{\rho}(\mu, \nu)=\gamma_{n}^{m} \delta_{n, n^{\prime}} \delta m, m^{\prime},
$$

with respect to the weight function

$$
l_{\rho}(\mu, \nu)=\frac{1}{\sqrt{\left(\rho^{2}-\mu^{2}\right)\left(\rho^{2}-\nu^{2}\right)}},
$$

where

$$
d s_{\rho}(\mu, \nu)=h_{\mu} h_{\nu} d \mu d \nu
$$

defines the differential surface element on the ellipsoid. Actually the weight function $l_{\rho}$ on any ellipsoid is a constant multiple of the support function. The constant $\gamma_{n}^{m}$ is the normalisation constant of the surface harmonic $S_{n}^{m}$.

Any smooth function $f$ defined over $S_{\rho}$ has the expansion

$$
f(\mu, \nu)=\sum_{n=0}^{\infty} \sum_{m=1}^{2 n+1} c_{n}^{m} S_{n}^{m}(\mu, \nu),
$$

with coefficients $c_{n}^{m}$ given by

$$
c_{n}^{m}=\frac{1}{\gamma_{n}^{m}} \oint_{S_{\rho}} f(\mu, \nu) S_{n}^{m}(\mu, \nu) l_{\rho}(\mu, \nu) d s_{\rho}(\mu, \nu) .
$$

The harmonics $S_{n}^{m}(\mu, \nu)$ over the surface of the ellipsoid correspond to the harmonics $Y_{n}^{m}(\theta, \phi)$ over the surface of the sphere. 
In the sequel we write down explicitly the harmonics of degree less than or equal to two.

For $n=0$, the Lamé function $E_{0}^{1}(x)=1$, and the ellipsoidal harmonic $\mathbb{E}_{0}^{1}(\rho, \mu, \nu)=1$, and

$$
\mathbb{F}_{0}^{1}(\rho, \mu, \nu)=\int_{\rho}^{\infty} \frac{d x}{\sqrt{\left(x^{2}-h_{3}^{2}\right)\left(x^{2}-h_{2}^{2}\right)}} .
$$

For $n=1$, we have the Lamé functions

$$
\begin{aligned}
& E_{1}^{1}(x)=x, \\
& E_{1}^{2}(x)=\sqrt{\left|x^{2}-h_{3}^{2}\right|}, \\
& E_{1}^{3}(x)=\sqrt{\left|x^{2}-h_{2}^{2}\right|},
\end{aligned}
$$

and the ellipsoidal harmonics

$$
\begin{aligned}
& \mathbb{E}_{1}^{1}(\rho, \mu, \nu)=\rho \mu \nu=h_{2} h_{3} x_{1}, \\
& \mathbb{E}_{1}^{2}(\rho, \mu, \nu)=\sqrt{\rho^{2}-h_{3}^{2}} \sqrt{\mu^{2}-h_{3}^{2}} \sqrt{h_{3}^{2}-\nu^{2}}=h_{1} h_{3} x_{2}, \\
& \mathbb{E}_{1}^{3}(\rho, \mu, \nu)=\sqrt{\rho^{2}-h_{2}^{2}} \sqrt{h_{2}^{2}-\mu^{2}} \sqrt{h_{2}^{2}-\nu^{2}}=h_{1} h_{2} x_{3},
\end{aligned}
$$

as well as

$$
\begin{aligned}
& \mathbb{F}_{1}^{1}(\rho, \mu, \nu)=3 \mathbb{E}_{1}^{1}(\rho, \mu, \nu) \int_{\rho}^{\infty} \frac{d x}{x^{2} \sqrt{\left(x^{2}-h_{3}^{2}\right)\left(x^{2}-h_{2}^{2}\right)}} \\
& \mathbb{F}_{1}^{2}(\rho, \mu, \nu)=3 \mathbb{E}_{1}^{2}(\rho, \mu, \nu) \int_{\rho}^{\infty} \frac{d x}{\left(x^{2}-h_{3}^{2}\right) \sqrt{\left(x^{2}-h_{3}^{2}\right)\left(x^{2}-h_{2}^{2}\right)}} \\
& \mathbb{F}_{1}^{3}(\rho, \mu, \nu)=3 \mathbb{E}_{1}^{3}(\rho, \mu, \nu) \int_{\rho}^{\infty} \frac{d x}{\left(x^{2}-h_{2}^{2}\right) \sqrt{\left(x^{2}-h_{3}^{2}\right)\left(x^{2}-h_{2}^{2}\right)}}
\end{aligned}
$$

For $n=2$, we have the Lamé functions

$$
\begin{aligned}
& E_{2}^{1}(x)=\left(x^{2}+\Lambda-\alpha_{1}^{2}\right), \\
& E_{2}^{2}(x)=\left(x^{2}+\Lambda^{\prime}-\alpha_{1}^{2}\right), \\
& E_{2}^{3}(x)=x \sqrt{\left|x^{2}-h_{3}^{2}\right|}, \\
& E_{2}^{4}(x)=x \sqrt{\left|x^{2}-h_{2}^{2}\right|}, \\
& E_{2}^{5}(x)=\sqrt{\left|x^{2}-h_{2}^{2}\right|} \sqrt{\left|x^{2}-h_{3}^{2}\right|},
\end{aligned}
$$

where $\Lambda$ and $\Lambda^{\prime}$ are the roots of the quadratic equation

$$
\sum_{i=1}^{3} \frac{1}{\Lambda-\alpha_{i}^{2}}=0 .
$$


We also have the ellipsoidal harmonics

$$
\begin{aligned}
\mathbb{E}_{2}^{1}(\rho, \mu, \nu) & =\left(\rho^{2}+\Lambda-\alpha_{1}^{2}\right)\left(\mu^{2}+\Lambda-\alpha_{1}^{2}\right)\left(\nu^{2}+\Lambda-\alpha_{1}^{2}\right) \\
& =\left(\Lambda-\alpha_{1}^{2}\right)\left(\Lambda-\alpha_{2}^{2}\right)\left(\Lambda-\alpha_{3}^{2}\right)\left[\sum_{i=1}^{3} \frac{x_{i}^{2}}{\Lambda-\alpha_{i}^{2}}+1\right] \\
\mathbb{E}_{2}^{2}(\rho, \mu, \nu) & =\left(\rho^{2}+\Lambda^{\prime}-\alpha_{1}^{2}\right)\left(\mu^{2}+\Lambda^{\prime}-\alpha_{1}^{2}\right)\left(\nu^{2}+\Lambda^{\prime}-\alpha_{1}^{2}\right) \\
& =\left(\Lambda^{\prime}-\alpha_{1}^{2}\right)\left(\Lambda^{\prime}-\alpha_{2}^{2}\right)\left(\Lambda^{\prime}-\alpha_{3}^{2}\right)\left[\sum_{i=1}^{3} \frac{x_{i}^{2}}{\Lambda^{\prime}-\alpha_{i}^{2}}+1\right] \\
\mathbb{E}_{2}^{3}(\rho, \mu, \nu) & =\rho \mu \nu \sqrt{\left(\rho^{2}-h_{3}^{2}\right)} \sqrt{\left(\mu^{2}-h_{3}^{2}\right)} \sqrt{\left(h_{3}^{2}-\nu^{2}\right)} \\
& =h_{1} h_{2} h_{3}^{2} x_{1} x_{2}, \\
\mathbb{E}_{2}^{4}(\rho, \mu, \nu) & =\rho \mu \nu \sqrt{\left(\rho^{2}-h_{2}^{2}\right)} \sqrt{\left(h_{2}^{2}-\mu^{2}\right)} \sqrt{\left(h_{2}^{2}-\nu^{2}\right)} \\
& =h_{1} h_{2}^{2} h_{3} x_{1} x_{3}, \\
\mathbb{E}_{2}^{5}(\rho, \mu, \nu) & =\sqrt{\left(\rho^{2}-h_{3}^{2}\right)} \sqrt{\left(\mu^{2}-h_{3}^{2}\right)} \sqrt{\left(h_{3}^{2}-\nu^{2}\right)} \sqrt{\left(\rho^{2}-h_{2}^{2}\right)} \sqrt{\left(h_{2}^{2}-\mu^{2}\right)} \sqrt{\left(h_{2}^{2}-\nu^{2}\right)} \\
& =h_{1}^{2} h_{2} h_{3} x_{2} x_{3},
\end{aligned}
$$

and

$$
\begin{aligned}
\mathbb{F}_{2}^{1}(\rho, \mu, \nu) & =5 \mathbb{E}_{2}^{1}(\rho, \mu, \nu) \int_{\rho}^{\infty} \frac{d x}{\left(x^{2}+\Lambda-\alpha_{1}^{2}\right)^{2} \sqrt{\left(x^{2}-h_{3}^{2}\right)\left(x^{2}-h_{2}^{2}\right)}}, \\
\mathbb{F}_{2}^{2}(\rho, \mu, \nu) & =5 \mathbb{E}_{2}^{2}(\rho, \mu, \nu) \int_{\rho}^{\infty} \frac{d x}{\left(x^{2}+\Lambda^{\prime}-\alpha_{1}^{2}\right)^{2} \sqrt{\left(x^{2}-h_{3}^{2}\right)\left(x^{2}-h_{2}^{2}\right)}} \\
\mathbb{F}_{2}^{3}(\rho, \mu, \nu) & =5 \mathbb{E}_{2}^{3}(\rho, \mu, \nu) \int_{\rho}^{\infty} \frac{d x}{x^{2}\left(x^{2}-h_{3}^{2}\right) \sqrt{\left(x^{2}-h_{3}^{2}\right)\left(x^{2}-h_{2}^{2}\right)}}, \\
\mathbb{F}_{2}^{4}(\rho, \mu, \nu) & =5 \mathbb{E}_{2}^{4}(\rho, \mu, \nu) \int_{\rho}^{\infty} \frac{d x}{x^{2}\left(x^{2}-h_{2}^{2}\right) \sqrt{\left(x^{2}-h_{3}^{2}\right)\left(x^{2}-h_{2}^{2}\right)}}, \\
\mathbb{F}_{2}^{5}(\rho, \mu, \nu) & =5 \mathbb{E}_{2}^{5}(\rho, \mu, \nu) \int_{\rho}^{\infty} \frac{d x}{\left(x^{2}-h_{3}^{2}\right)\left(x^{2}-h_{2}^{2}\right) \sqrt{\left(x^{2}-h_{3}^{2}\right)\left(x^{2}-h_{2}^{2}\right)}}
\end{aligned}
$$

\section{REFERENCES}

[1] G. K. Batchelor, An Introduction to Fluid Dynamics, Cambridge University Press, Cambridge, 1967. MR1744638 (2000j:76001)

[2] A. Bounaïm, S. Holm, Wen Chen and Å. Ødegård, Detectability of Breast Lesions with CARI ultrasonography using a bioacoustic computational approach, Comput. Math. Appl., 54, 96-106, 2007. MR2332779 (2008d:92028)

[3] L. I. Cardenas-Navia, R. A. Richardson, and M. W. Dewhirst, Targeting the molecular effects of a hypoxic tumor microenvironment. Front Biosci. 2007 May 1;12:4061-78. Review.

[4] M. A. J. Chaplain and B. D. Sleeman. Modelling the growth of solid tumours and incorporating a method for their classification using nonlinear elasticity theory, J. Math. Biol., 31, 431-473, 1993. MR 1229444

[5] Q. Y. Gong, P. R. Eldridge, A. R. Brodbelt, et al., Quantification of Tumour Response to Radiotherapy, The British J. Radiology, 77, 405-413, 2004.

[6] H. P. Greenspan, Models for the growth of a solid tumour, Stud. Appl. Math, 52, 317-340, 1972. 
[7] H. P. Greenspan, On the growth and stability of cell cultures and solid tumours, J. Theor. Biol, 56, 229-242, 1976. MR0429164(55:2183)

[8] G. Helminger, P. A. Netti, H. C. Lichtenbeld, R. J. Melder and R. K. Jain (1997), Solid stress inhibits the growth of multicellular tumor spheroids, Nat. Biotechnology, 15, 778-783.

[9] E. W. Hobson, The theory of spherical and ellipsoidal harmonics, Cambridge University Press, 1931.

[10] J. Folkman (1971), Tumour angiogenesis: therapeutic implications. New England J. Medicine, 285, $1182-1186$.

[11] J. L. Gevertz, G. T. Gillies, and S. Torquato (2008), Simulating tumor growth in confined heterogeneous environments. Phys. Biol., 5, 1-10.

[12] P. F. Jones and B. D. Sleeman, Mathematical Modelling of Avascular and Vascular Tumour Growth. Advanced Topics in Scattering and Biomedical Engineering, Eds. A. Charalambopoulos, D. I. Fotiadis and D. Poyzos, World Scientific, pp. 305-331 (2008). MR2433048 (2009m:92029)

[13] Z. K. Otrock, R. A. Mahfouz, J. A. Makarem and A. I. Shamseddine. Understanding the biology of angiogenesis: review of the most important molecular mechanisms. Blood Cells Mol. Dis. 2007 Sep-Oct;39(2):212-20. Epub 2007 Jun 6. Review.

[14] M. J. Plank and B. D. Sleeman (2003), Tumour-induced Angiogenesis: A Review. J. Theor. Medicine, 5, 137-153.

[15] T. Roose, S. J. Chapman and P. K. Maini, Mathematical Models of Avascular Tumor Growth. SIAM Review, 49, 179-208, 2007. MR2327053 (2008d:92013)

[16] K. Shcors and G. Evan, Tumor angiogenesis: cause or consequence of cancer? Cancer Res. 2007 Aug 1;67(15):7059-61. Review.

[17] R. Xu, G. C. Anagnostopoulos and D. C. Wunsch, Multiclass Cancer Classification Using Semisupervised Ellipsoid ARTMAP and particle Swarm Optimization with Gene Expression Data. IEEE/ACM Trans. Comp. Biol. Bioinformatics, 4, 65-77, 2007. 